\title{
Impact of operating temperature on the electrical and magnetic properties of the bottom-pinned perpendicular magnetic tunnel junctions
}

Y. C. Wu, W. Kim, S. Rao, K. Garello, S. Van Beek, S. Couet, E. Liu, J. Swerts, S. Kundu, L. Souriau, F. Yasin, D. Crotti, J. K. Jochum, M. J. Van Bael, J. Van Houdt, G. Groeseneken, and G. S. Kar

Citation: Appl. Phys. Lett. 113, 142405 (2018); doi: 10.1063/1.5042028

View online: https://doi.org/10.1063/1.5042028

View Table of Contents: http://aip.scitation.org/toc/apl/113/14

Published by the American Institute of Physics

\section{Articles you may be interested in}

Micromagnetic simulation of spin torque ferromagnetic resonance in nano-ring-shape confined magnetic tunnel junctions

Applied Physics Letters 113, 142406 (2018); 10.1063/1.5042447

A non-collinear double $\mathrm{MgO}$ based perpendicular magnetic tunnel junction

Applied Physics Letters 113, 022403 (2018); 10.1063/1.5038060

High performance perpendicular magnetic tunnel junction with Co/Ir interfacial anisotropy for embedded and standalone STT-MRAM applications

Applied Physics Letters 112, 092402 (2018); 10.1063/1.5018874

Measurements of interlayer exchange coupling of Pt in Py|Pt|Py system

Applied Physics Letters 113, 142401 (2018); 10.1063/1.5050935

Spin-orbit torques in high-resistivity-W/CoFeB/MgO

Applied Physics Letters 112, 192408 (2018); 10.1063/1.5027855

Current-induced four-state magnetization switching by spin-orbit torques in perpendicular ferromagnetic trilayers Applied Physics Letters 113, 112406 (2018); 10.1063/1.5034380

\section{Lake Shore CRYOTRONICS}

\section{Sensors, Controllers, Monitors} from the world leader in cryogenic thermometry 


\title{
Impact of operating temperature on the electrical and magnetic properties of the bottom-pinned perpendicular magnetic tunnel junctions
}

\author{
Y. C. Wu ${ }^{1,2}$ W. Kim, ${ }^{1, a)}$ S. Rao, ${ }^{1}$ K. Garello, ${ }^{1}$ S. Van Beek,,${ }^{1,2}$ S. Couet, ${ }^{1}$ E. Liu, ${ }^{1,2}$ \\ J. Swerts, ${ }^{1}$ S. Kundu, ${ }^{1}$ L. Souriau, ${ }^{1}$ F. Yasin, ${ }^{1}$ D. Crotti, ${ }^{1}$ J. K. Jochum, ${ }^{3}$ M. J. Van Bael, ${ }^{3}$ \\ J. Van Houdt, ${ }^{1,2}$ G. Groeseneken, ${ }^{1,2}$ and G. S. Kar ${ }^{1}$ \\ ${ }^{1}$ IMEC, Kapeldreef 75, 3001 Heverlee, Belgium \\ ${ }^{2}$ Department of Electrical Engineering, KU Leuven, Kasteelpark Arenberg 10, 3001 Heverlee, Belgium \\ ${ }^{3}$ Laboratory of Solid State Physics and Magnetism, KU Leuven, Celestijnenlaan 200D, 3001 Heverlee, \\ Belgium
}

(Received 29 May 2018; accepted 20 September 2018; published online 4 October 2018)

\begin{abstract}
Analogous device parameters in both the parallel (P) and anti-parallel (AP) states ensure a symmetric spin-transfer-torque magnetic random-access memory operation scheme. In this study, however, we observe an increasing asymmetry in the performance metrics with operating temperature of the bottom-pinned perpendicular magnetic tunnel junction (p-MTJ) devices. A temperature-dependent increase in the contribution of the stray field is observed in the tunneling magnetoresistance loop analysis. The switching current for P-to-AP decreases by $30 \%$ in the thermally activated switching regime by increasing the temperature from $300 \mathrm{~K}$ to $400 \mathrm{~K}$, while it remains similar for AP-to-P. In addition, with the same temperature range, the thermal stability factor for the P state decreases $20 \%$ more than that for the AP state. We attribute those observations to the increase in the overcompensation of the stray field from the synthetic anti-ferromagnet structure. Saturation magnetization $\left(M_{S}\right)$ of the $[\mathrm{Co} / \mathrm{Pt}]_{\mathrm{x}}$-based multilayers is much less affected by temperature $\left[M_{S}(400 \mathrm{~K}) / M_{S}(300 \mathrm{~K})=97 \%\right]$ compared to that of the CoFeB-based multilayers (88\%). Such an impact can be more severe during the electrical switching process due to the Joule heating effect. These results suggest that, to understand and to evaluate the performance in a wide range of temperatures, it is crucial to consider the contribution of the entire magnetic components in the p-MTJ stack. Published by AIP Publishing.

https://doi.org/10.1063/1.5042028
\end{abstract}

Spin-transfer-torque magnetic random-access memory (STT-MRAM) with a perpendicular magnetic tunnel junction (p-MTJ) is a promising technology for future cache and embedded non-volatile memory applications due to its complementary metal-oxide-semiconductor (CMOS) compatibility, fast operation speed, high endurance, and long data retention. ${ }^{1-4}$ Potentially, it is applicable to versatile electronic equipment, e.g., mobile phones, computers, and automotive applications. The operating temperature required for such applications is often as wide as $235 \mathrm{~K}-355 \mathrm{~K}$ and can be higher than $400 \mathrm{~K}$ for automotive uses. ${ }^{5}$ Basic device functions such as reading, writing, and data retention of the p-MTJ devices vary with operating temperature. ${ }^{6}$ The fundamental reasons for the variation are the change in the intrinsic ferromagnetic properties of the free layer (FL) and the transport properties through the $\mathrm{MgO}$ tunnel barrier. ${ }^{7,8}$ Apart from these, the magneto-static fields generated from the adjacent magnetic layers, i.e., the reference layer (RL) and the hard layer (HL), act as additional factors to change the p-MTJ performance. By coupling the RL and the $\mathrm{HL}$ anti-ferromagnetically to form a synthetic anti-ferromagnet (SAF), the net magneto-static field is compensated. The over/under-compensated field is referred to as the stray field $\left(H_{\text {stray }}\right)$. Its sign and magnitude are crucial to the device operation, and it should be controlled by stack and process engineering. ${ }^{9,10}$ Previous reports investigate the $\mathrm{p}$ MTJ device performance at various operating temperatures in terms of the variation in the FL intrinsic properties, ${ }^{6,11}$ and the

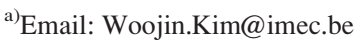

potential change in SAF properties are, however, not considered as additional factors to influence the high temperature performance. In this study, we discuss the temperature-dependent ( $T$-dependent) change in the stray field $\left[H_{\text {stray }}(T)\right]$ and its origin, thereby correlating the impact of the $H_{\text {stray }}(T)$ with the asymmetric behavior in the p-MTJ device performance metrics, e.g., the switching current $\left(I_{s w}\right)$ and the thermal stability factors $(\Delta)$, at different operating temperatures.

A p-MTJ stack composed of bottom-electrode/seed/ $\mathrm{HL}(4.6) / \mathrm{Ru}(0.4) / \mathrm{RL}(2.2) / \mathrm{MgO}(1) / \mathrm{FL}(2.6) / \mathrm{MgO}(0.5) / \mathrm{Ta} / \mathrm{Ru} /$ top-electrode is deposited on a $300 \mathrm{~mm}$ thermally oxidized $\mathrm{Si}(100)$ wafer in a Canon-ANELVA EC7800 cluster tool. Numbers in parentheses are the nominal thicknesses in $\mathrm{nm}$. After deposition, the film is annealed at $375^{\circ} \mathrm{C}$ for $30 \mathrm{~min}$ in vacuum under the out-of-plane magnetic field of $20 \mathrm{kOe}$. The RL is built up with a $\mathrm{Co} / \mathrm{spacer} / \mathrm{CoFeB}$ multilayer in general, and it is anti-ferromagnetically coupled to the $[\mathrm{Co} /$ $\mathrm{Pt}]_{\mathrm{x}}$-based $\mathrm{HL}$ to form a SAF structure. The dual-MgO FL consists of $\mathrm{CoFeB}(1.3) / \mathrm{Ta}(0.4) / \mathrm{CoFeB}(0.9)$, in which the two $\mathrm{CoFeB}$ layers are ferromagnetically coupled by the $\mathrm{Ta}$ spacer. ${ }^{12}$ The stack is then patterned into p-MTJ pillars by $193 \mathrm{~nm}$ immersion lithography and ion beam etching (IBE). The resistance-area $(R A)$ product which is around $10.5 \Omega \mu \mathrm{m}^{2}$ is estimated through multiple $500 \mathrm{~nm}$ devices. The electrical dimension $(e-C D)$ of the other devices is then derived by $e-C D=\left[\frac{4}{\pi}\left(R_{p, 500 \mathrm{~nm}} \times A_{500 \mathrm{~nm}} / R_{p}\right)\right]^{1 / 2}$, where $R_{p, 500 \mathrm{~nm}}$ and $A_{500 \mathrm{~nm}}$ are the parallel resistance and the area of the $500 \mathrm{~nm}$ devices. Devices in discussion have $e-C D=50 \mathrm{~nm}$ in median. An additional stack of $\mathrm{Si} / \mathrm{SiO}_{2} / \mathrm{seed} /[\mathrm{Co} / \mathrm{Pt}]_{\mathrm{x}}(4.6) /$ 
$\mathrm{Ru}(50)$ is prepared with the same deposition and annealing conditions as the p-MTJ stack to compare the magnetic properties of the stand-alone $[\mathrm{Co} / \mathrm{Pt}]_{\mathrm{x}}$ with the HL. Magnetic moments of these blanket stacks are quantified by a LOT-Quantum Design MPMS3 Superconducting Quantum Interference Device Vibrating Sample Magnetometer (SQUID-VSM) on the $3 \times 3 \mathrm{~mm}^{2}$ samples.

Stray fields from the SAF structure are estimated by examining the offsets in the tunneling magnetoresistance (TMR) loops. Each device is evaluated from 25 iterations. Figure 1(a) shows the average TMR loops of an example device, and the dotted lines are the offsets which indicate the $H_{\text {stray }}$. These $H_{\text {stray }}$ values are quantified from the average switching fields $H_{s w}=H_{c} \pm H_{\text {stray }}$, where $H_{c}$ is the FL coercivity. The negative sign of $H_{\text {stray }}$ indicates that the FL favors in the anti-parallel (AP) state than in the parallel (P) state. Figure 1(b) shows the statistical TMR variation of 120 devices as a function of operating temperature, observed in Fig. 1(a). It is a general behavior, and its dependence is also similar in the literature. ${ }^{6}$ In addition, we observe an increase in the $H_{\text {stray }}$ as the operating temperature is increased in Fig. 1(a). Since not only does the $H_{c}$ of the FL decrease but also the $H_{\text {stray }}$ increases with temperature, the $H_{\text {stray }} / H_{c}$ ratio increases significantly, as shown in Fig. 1(c). The $H_{\text {stray }} / H_{c}$ ratio is often taken as an example parameter to compare and analyze with the electrical switching metrics. Its increase is contributed by both increasing $H_{\text {stray }}$ and decreasing $H_{c}$ in our devices. The origin and the magnitude of the $T$-dependent stray field $H_{\text {stray }}(T)$ will follow in the discussion on the result of SQUID-VSM measurements.

To extend the observations in $H_{\text {stray }}(T)$, we perform electrical switching on 10 randomly selected devices from those in Fig. 1. e-CD of these selected devices is $46 \mathrm{~nm}$ in median. For each device, 10 switching events for both polarities are carried out with the pulse width $\left(t_{p}\right)$ ranging from

(a)
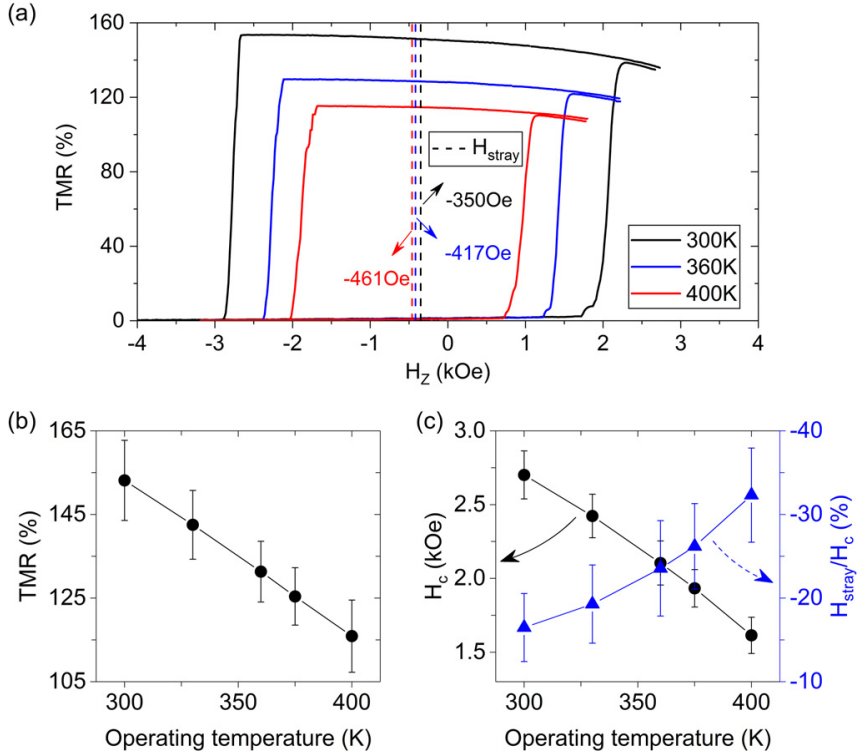

FIG. 1. (a) TMR loops of an example p-MTJ device from 25 iterations at different operating temperatures. Dotted lines indicate stray fields of the loops. (b) TMR of 120 devices as a function of operating temperature. (c) (Black circle) Coercivities estimated from the average TMR loop at different temperatures. (Blue triangle). Ratio of the stray fields to the coercivities at different temperatures. Error bars indicate the standard deviation.
$100 \mathrm{~ns}$ to $1 \mathrm{~ms}$. Figure 2(a) summarizes the switching currents $\left(I_{s w}\right)$ from all switching events at various temperatures and pulse widths. $I_{s w}$ of P-to-AP switching $\left(I_{s w, P \text {-to-AP }}\right)$ reduces substantially with operating temperature, while the variation is marginal for $I_{s w}$ of AP-to-P switching $\left(I_{s w, A P-t o-P}\right)$. An example of such asymmetric behavior at $100 \mathrm{~ns}$ pulse is also summarized in the inset of Fig. 2(b). $I_{s w, A P-t o-P}$ remains approximately constant $(112 \mu \mathrm{A}$ in median) within the explored temperature range. On the other hand, $I_{s w, P \text {-to-AP }}$ drastically reduces from $100 \mu \mathrm{A}$ to $69 \mu \mathrm{A}$. We further confirm the asymmetric behavior with extended statistics on an additional batch of 80 devices, of which $e-C D$ is $52 \mathrm{~nm}$ in median. The difference in switching currents originates from the size variation of devices and batches, but the asymmetric behavior is still consistent. We attribute these asymmetric phenomena to the increasing $H_{\text {stray }} /$ $H_{c}$ ratio, which influences the stability of both states in an opposite manner. These switching currents are subsequently modeled based on the macro-spin model ${ }^{13-16}$

$$
\begin{gathered}
I_{c 0}=2 e \alpha M_{S} H_{k, e f f} V / \hbar \eta, \\
I_{s w}=I_{c 0}\left(1-\frac{1}{\Delta} \ln \left(\frac{t_{p}}{\tau_{0}}\right)\right),
\end{gathered}
$$

(a)

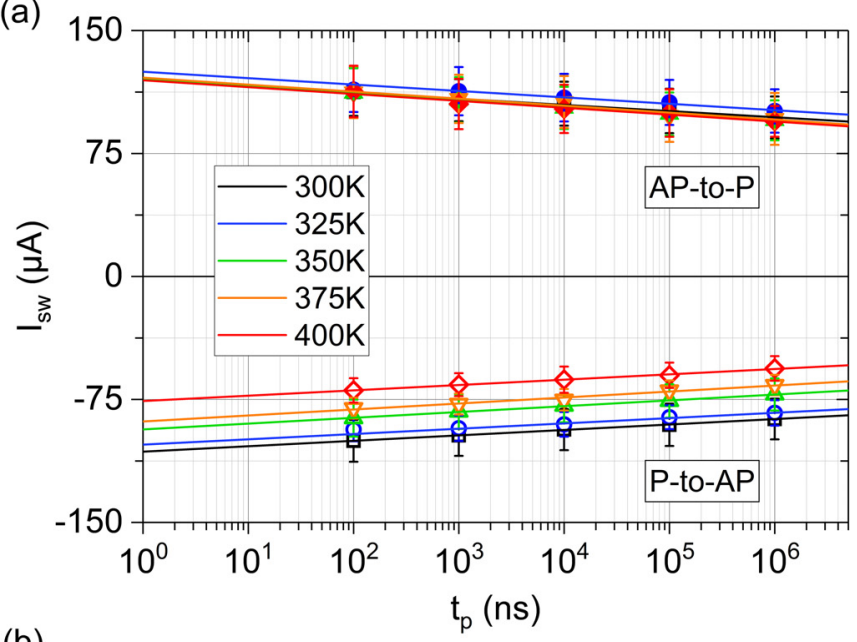

(b)

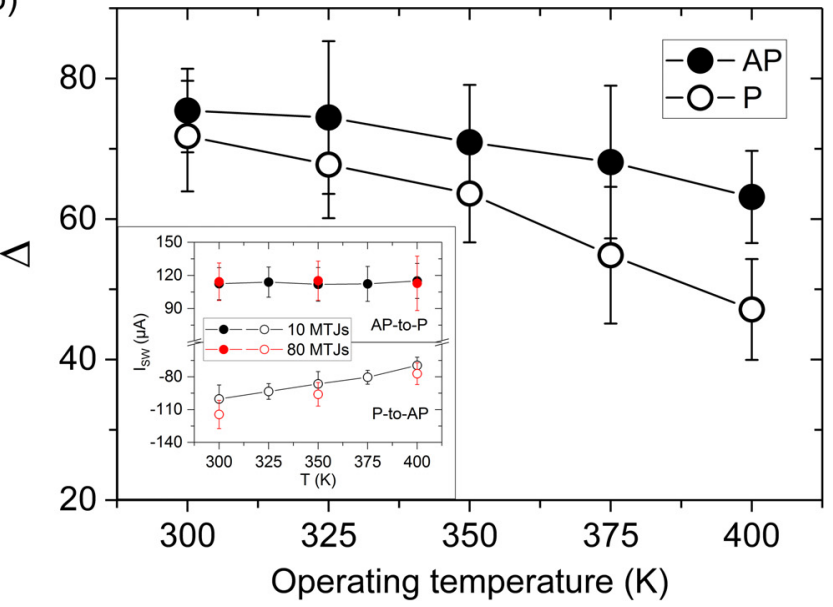

FIG. 2. (a) Switching currents of 10 random selected devices as a function of pulse widths at various temperatures. The solid (open) symbols denote AP-to-P (P-to-AP) switching. For each pulse width, 10 switching events are carried out per device. (b) Thermal stability factors extracted with Eq. (2) at various temperatures. Inset: Switching currents of (Black symbols) 10 devices in (a) and (Red symbols) another batch of 80 similar devices, at $100 \mathrm{~ns}$ pulse at $300-400 \mathrm{~K}$. Error bars indicate the standard deviation. 
where $\alpha$ is the damping constant, $M_{S}$ and $V$ are the saturation magnetization and volume of the FL, $\hbar$ is the reduced Planck constant, $\eta$ is the spin torque efficiency, and $\tau_{0}$ is the attempt rate $(1 \mathrm{~ns})$. The FL effective anisotropy field $H_{k, \text { eff }}$ includes the intrinsic magnetic anisotropy field, the demagnetizing field, and the external field $\left(H_{k, e f f}=H_{k, i}+H_{\text {demag }}+H_{\text {ext }}\right)$. The thermal stability factor $(\Delta)$ is the energy barrier $\left(E_{b} / k_{B} T=M_{S, F L} H_{k, \text { eff }} / 2 k_{B} T\right)$ between both states. Due to the increasing thermal energy which reduces $M_{S, F L}$ and $H_{k, e f f}{ }^{7}$ both states become less stable at elevated temperatures. However, as discussed in Fig. 1, the increase in $H_{\text {stray }}(T)$ stabilizes the AP state and destabilizes the $\mathrm{P}$ state. In the existence of $H_{\text {stray }}$, an additional term $+H_{\text {stray }}$ $\left(-H_{\text {stray }}\right)$, which acts as an external field to the FL, has to be included in $H_{k, \text { eff }}$ for the AP (P) state. Consequently, the influence of temperature is compensated for $\Delta_{\mathrm{AP}}$, while it becomes more significant for $\Delta_{\mathrm{P}}$. In median, $\Delta_{\mathrm{AP}}$ reduces from 75 at $300 \mathrm{~K}$ to 63 at $400 \mathrm{~K}$, and $\Delta_{\mathrm{P}}$ drops more significantly from 71 to 47 , as shown in Fig. 2(b).

Next, we discuss the origin of $H_{\text {stray }}(T)$. Magnetic moments of the p-MTJ blanket stacks are measured as a function of the out-of-plane magnetic field (M-H loops) with SQUID-VSM at different sample temperatures, as shown in Fig. 3(a). The magnetic field is swept between $\pm 60 \mathrm{kOe}$ and
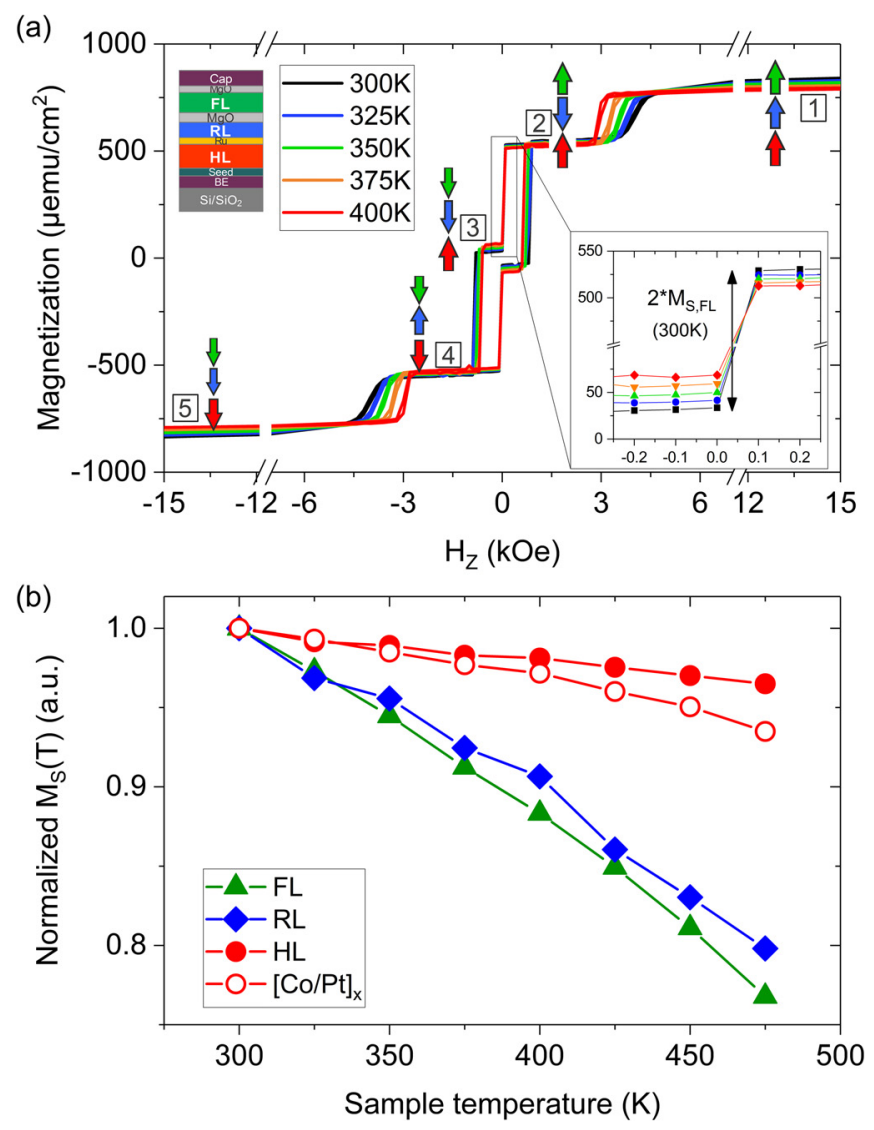

FIG. 3. (a) SQUID-VSM measurements of the magnetic moments as a function of out-of-plane magnetic field (M-H loops) quantified on $3 \times 3 \mathrm{~mm}^{2}$ samples at various sample temperatures. Labels $1-5$ and the nearby cartoons indicate the five different magnetization configurations at different magnetic field stages. Inset: Detailed FL switching region (from 2 to 3 ) at various sample temperatures, where the magnetization of the FL is extracted. The difference is equal to $2 * \mathrm{M}_{\mathrm{S}, \mathrm{FL}}$. (c) Normalized saturation magnetization $\left[M_{S}(T) / M_{S}(300 \mathrm{~K})\right]$ as a function of sample temperature. Each data point is estimated as an average of five samples. we show the result within $\pm 15 \mathrm{kOe}$ for better visualization. Magnetic moments are recorded at every 100 Oe within the range of $\pm 5 \mathrm{kOe}$. Steep changes in the curves correspond to the reversals of the magnetic layers. At stages 1 , the magnetic moment is saturated which gives $M_{S, \text { Saturation }}$. From 1 to 2, the RL switches as it is anti-ferromagnetically coupled to the HL. The coupling strength is around $5 \mathrm{kOe}$ at $300 \mathrm{~K}$. Stage 2 to 3 is the FL switch, and the details of this switch are shown in the inset of Fig. 3(a). From stage 3 to 4, the RL and the HL reverse simultaneously (SAF flip) as a collective behavior of anti-ferromagnetically coupled magnetic layers. Finally, the stack saturates in the negative direction as the external field exceeds the coupling field to reverse the RL (4 to 5). The magnetic configurations are sketched in cartoons. At each temperature, we evaluate the $M_{S, R L}$ (1) to 2) and the $M_{S, F L}$ (2 to 3). Detailed evaluation of the FL magnetic moments is illustrated in the inset of Fig. 3(a) as an example. $M_{S, H L}$ is subsequently quantified by deducting $M_{S, R L}$ and $M_{S, F L}$ from $M_{S, \text { Saturation. }}$. At room temperature (RT), we extract $M_{S, F L}, M_{S, R L}$, and $M_{S, H L}$ as $964 \mathrm{emu} / \mathrm{cm}^{3}$, $709 \mathrm{emu} / \mathrm{cm}^{3}$, and $950 \mathrm{emu} / \mathrm{cm}^{3}$, respectively. These saturation magnetizations are derived by considering the entire thickness of all those layers, including the non-magnetic spacers. $M_{S, F L}(R T)$ is consistent with the reported values in similar CoFeB-based dual $\mathrm{MgO}$ free layers, ${ }^{12,17}$ while $M_{S, R L}(R T)$ is estimated to be smaller owing to a higher boron content and a thicker spacer. The temperature sensitivity of these three layers is summarized in Fig. 3(b) by averaging the $T$-dependence of $M_{S, F L}, M_{S, R L}$, and $M_{S, H L}$ from five samples. The two CoFeB-based layers, the FL and the RL, have similar $T$-dependence, but the HL is observed to have clearly different temperature sensitivity from that of the FL and the RL. Compared with the measurements on the stand-alone $[\mathrm{Co} / \mathrm{Pt}]_{\mathrm{x}}$ samples, we confirm that such divergence is mostly an intrinsic behavior of the HL itself.

The $T$-dependence of the saturation magnetization is generally described by Bloch's law, ${ }^{18}$ with $M_{S}$ at $0 \mathrm{~K}\left[M_{S}(0)\right]$ and the Curie temperature $\left(T_{C}\right)$ as fitting parameters. $M_{S}(0)$ and $T_{C}$ are estimated to be $1142 \mathrm{emu} / \mathrm{cm}^{3}$ and $1032 \mathrm{~K}$ for the FL and $849 \mathrm{emu} / \mathrm{cm}^{3}$ and $1079 \mathrm{~K}$ for the RL. These values are consistent with other reports on similar CoFeB-based materials. ${ }^{7,19}$ However, fitting the present result of the HL with Bloch's law gives $T_{C}$ higher than that reported in other studies, ${ }^{20,21}$ and furthermore, higher than the pure $\mathrm{Fe}$ or Co. ${ }^{19}$ In the past few decades, $[\mathrm{Co} / \mathrm{Pt}]_{\mathrm{x}}$ multilayers have been studied for various points of interests, ${ }^{20-22}$ but their characteristics vary with different conditions such as thickness combinations of $\mathrm{Co}$ and $\mathrm{Pt}$, number of repeats, and deposition conditions. Moreover, Bloch's law deviates from practice if the studied temperature range is not sufficiently lower than $T_{C}$ of the materials, which might be the case of $[\mathrm{Co} / \mathrm{Pt}]_{\mathrm{x}}$-based multilayers since these materials often possess $T_{C}$ lower than $700 \mathrm{~K} \cdot{ }^{19,21}$ In this study, we consider the $T$-dependence of magnetization in the operating temperatures to describe the device operation. Thus, further detailed characterization of the $T$-dependence of $[\mathrm{Co} / \mathrm{Pt}]_{\mathrm{x}}$ multilayers is above the scope in our discussion, but it will be interesting to be followed by in-depth investigation, especially with the conditions compatible to be used in high-performance p-MTJs. 
Based on the SQUID data, we also estimate the relative change in the stray field $\Delta H_{\text {stray }}(\mathrm{T})\left(H_{\text {stray,@T. }}-H_{\text {stray,@300K }}\right)$ by micro-magnetic simulation using the Object Oriented MicroMagnetic Framework ${ }^{23}$ (OOMMF) project to compare with the experiments. In Fig. 4(a), we first plot $\Delta H_{\text {stray }}(\mathrm{T})$ from the experimental results of TMR loops as a function of operating temperature. Despite wide distribution of experimental data, the overall shift of $\Delta H_{\text {stray }}$ in the negative direction signifies the stronger stray field at higher temperatures. For the simulation, p-MTJ devices are built as a cylindrical pillar with $1 \times 1 \times 0.2 \mathrm{~nm}^{3}$ unit cells, of which the stack configuration is identical to our p-MTJ stack. With the experimental $M_{S, R L}(T)$ and $M_{S, H L}(T)$ obtained from Fig. 3 as input parameters, we extract the average $H_{\text {stray }}(T)$ generated from the SAF structure at the FL position. Due to the significant reduction in $M_{S, R L}(T)$ compared to $M_{S, H L}(T)$, simulation results confirm the similar $\Delta H_{\text {stray }}$ trend to the experiments, as shown in Fig. 4(b). Such a difference in the temperature sensitivity of $M_{S, R L}$ and $M_{S, H L}$ explains the origin of $T$ dependent offsets in the device TMR loops [Fig. 4(a)], the resultant increasing $H_{\text {stray }} / H_{c}$ ratio [Fig. 1(c)], and the consequent $T$-dependent asymmetry in $I_{s w}$ and $\Delta$ (Fig. 2). Furthermore, simulation suggests that $\Delta H_{\text {stray }}$ will be more considerable as the device scales down. To avoid such

(a)

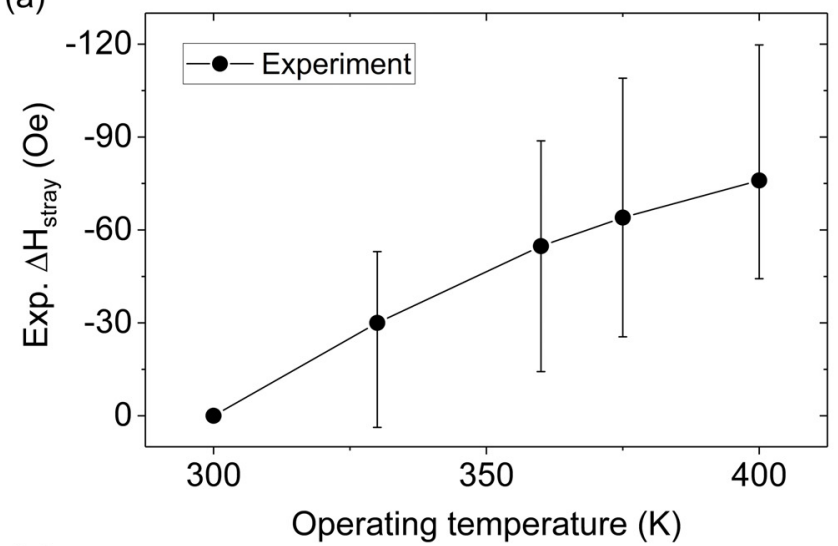

(b)

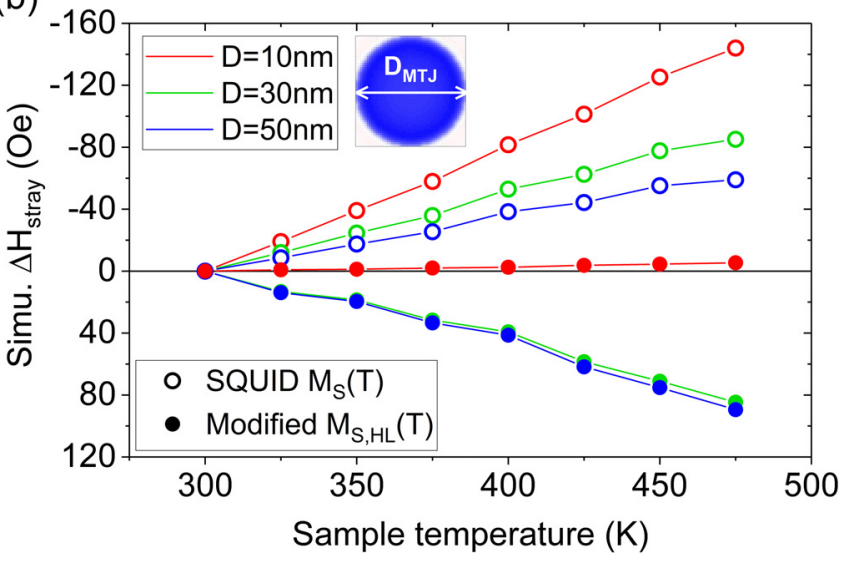

FIG. 4. (a) The relative change in the stray field $\left(H_{\text {stray,@T }}-H_{\text {stray,@300K }}\right)$ experimentally, estimated from TMR loops, with the error bars indicating the quartiles of the dataset. (b) Micromagnetic simulation (OOMMF) results of the relative change in stray fields. (Open symbols) Simulation results based on the SQUID $M_{S, R L}(T)$ and $M_{S, H L}(T)$. (Solid symbols) Assume that a HL material has a modified $M_{S}(T)$ trend which is the same as the RL in Fig. 3(b). Stray fields at each temperature are the average magneto-static field at the FL position.
$T$-dependent issues, modification of the temperature sensitivity can be a potential solution to minimize the $T$-dependent variation in the stray field. For example, we calculate $\Delta H_{\text {stray }}$ by assuming that the modified HL material has the same temperature sensitivity as the RL in Fig. 3(b), i.e., more sensitive to temperature than the experimental data, with $M_{S, H L}(R T)$ equal to $950 \mathrm{emu} / \mathrm{cm}^{3}$. Under these conditions, $\Delta H_{\text {stray }}(T)$ in all sizes shifts to the positive, as shown in Fig. 4(b). $\Delta H_{\text {stray }}(T)$ can be minimized to nearly zero for the $10 \mathrm{~nm}$ device in the temperature range of interest. However, in relatively large devices, $\Delta H_{\text {stray }}(T)$ is changed too much that even the sign is altered. According to these results, for the larger devices, a modified HL with temperature sensitivity different from both the experimental RL and HL can achieve zero $\Delta H_{\text {stray }}(T)$. These simulation results also show that the $T$ dependent stray field can be optimized for a targeted device size by modification of the temperature sensitivity.

Finally, we remark that the influence of $H_{\text {stray }}(T)$ can be more significant in current-induced switching than the observation in the TMR loops because of the Joule heating effect. In Ref. 24, the effective device temperature has been experimentally evaluated. With sufficiently long pulses, Joule heating can elevate the effective temperature by approximately $100 \mathrm{~K}$ or even higher during the pulse duration, depending on the applied voltage and current. ${ }^{24,25}$ In this case, following the trends in Figs. 3(b) and 4(a), additional $100 \mathrm{~K}$ increase in temperature will significantly increase $H_{\text {stray }}$. As an example, the SAF structure in our p-MTJ device at RT can exert nearly 80 Oe stronger stray field on the FL considering that the effective device temperature is $100 \mathrm{~K}$ higher than RT during the current-induced switching process. Asymmetry in all performance metrics can be more severe than expected, consequently.

In summary, we investigate the electrical and magnetic properties of the bottom-pinned p-MTJ at various operating temperatures. Stray fields from the TMR loops shift with increasing operating temperature in the AP-state-favored direction in our devices. Increasing $H_{\text {stray }}$ along with the reduction of the $H_{c}$ impacts the switching currents and the thermal stability factors asymmetrically. Such an increase in the stray field originates from the different $T$-dependent saturation magnetization between the HL and the RL materials, thereby adding extra variations to the device performance. These results suggest that in addition to the intrinsic FL properties, detailed studies on the $T$-dependent magnetic properties of the SAF structure are crucial to optimize the device operation parameters in a wide range of operating temperatures.

This work was supported by IMEC's Industrial Affiliation Program on MRAM devices and financially supported by the Research Foundation Flanders (FWO), the KU Leuven Concerted Research Action GOA/14/007, and the Hercules Foundation.

${ }^{1}$ J. C. Slonczewski, J. Magn. Magn. Mater. 159, L1 (1996).

${ }^{2}$ L. Thomas, G. Jan, J. Zhu, H. Liu, Y. J. Lee, S. Le, R. Y. Tong, K. Pi, Y.

J. Wang, D. Shen, R. He, J. Haq, J. Teng, V. Lam, K. Huang, T. Zhong, T. Torng, and P. K. Wang, J. Appl. Phys. 115, 172615 (2014).

${ }^{3}$ D. C. Worledge, G. Hu, D. W. Abraham, J. Z. Sun, P. L. Trouilloud, J. Nowak, S. Brown, M. C. Gaidis, E. J. O’Sullivan, and R. P. Robertazzi, Appl. Phys. Lett. 98, 022501 (2011). 
${ }^{4}$ S. Bhatti, R. Sbiaa, A. Hirohata, H. Ohno, S. Fukami, and S. N. Piramanayagam, Mater. Today 20, 530-548 (2017).

${ }^{5}$ See https://www.altera.com/products/common/temperature/ind-temp.html for more information about operating temperature; accessed 13 March 2018. ${ }^{6}$ C. Park, J. J. Kan, C. Ching, J. Ahn, L. Xue, R. Wang, A. Kontos, S. Liang, M. Bangar, H. Chen, S. Hassan, S. Kim, M. Pakala, and S. H. Kang, IEEE. Trans. Magn. 53(2), 3400104 (2017).

${ }^{7}$ J. G. Alzate, P. K. Amiri, G. Yu, P. Upadhyaya, J. A. Katine, J. Langer, B. Ocker, I. N. Krivorotov, and K. L. Wang, Appl. Phys. Lett. 104, 112410 (2014).

${ }^{8}$ S. Yuasa, A. Fukushima, H. Kubota, Y. Suzuki, and K. Ando, Appl. Phys. Lett. 89, 042505 (2006).

${ }^{9}$ Y. H. Wang, S. H. Huang, D. Y. Wang, K. H. Shen, C. W. Chien, K. M. Kuo, S. Y. Yang, and D. L. Deng, in IEEE International Electron Devices Meeting 2012, San Francisco, USA, 10 December-13 December 2012, pp. 29.2.1-29.2.4

${ }^{10}$ M. Yoshikawa, T. Kai, M. Amano, E. Kitagawa, T. Nagase, M. Nakayama, S. Takahashi, T. Ueda, T. Kishi, K. Tsuchida, S. Ikegawa, Y. Asao, H. Yoda, Y. Fukuzumi, K. Nagahara, H. Numata, H. Hada, N. Ishiwata, and S. Tahara, J. Appl. Phys. 97, 10P508 (2005).

${ }^{11}$ J. Igarashi, J. Llandro, H. Sato, F. Matsukura, and H. Ohno, Appl. Phys. Lett. 111, 132407 (2017).

${ }^{12}$ S. Couet, J. Swerts, S. Mertens, T. Lin, Y. Tomczak, E. Liu, B. Douhard, S. Van Elshocht, A. Furnemont, and G. S. Kar, IEEE. Magn. Lett. 7, 3103004 (2016).

${ }^{13}$ Z. Li and S. Zhang, Phys. Rev. B 69, 134416 (2004).
${ }^{14}$ H. Sato, M. Yamanouchi, K. Miura, S. Ikeda, H. D. Gan, K. Mizunuma, R. Koizumi, F. Matsukura, and H. Ohno, Appl. Phys. Lett. 99, 042501 (2011).

${ }^{15}$ L. Tillie, E. Nowak, R. C. Sousa, M. C. Cyrille, B. Delaet, T. Magis, A. Persico, J. Langer, B. Ocker, I. L. Prejbeanu, and L. Perniola, in IEEE International Electron Devices Meeting 2016, San Francisco, USA, 3 December-7 December 2016, pp. 27.3.1-27.3.4.

${ }^{16}$ R. H. Koch, J. A. Katine, and J. Z. Sun, Phys. Rev. Lett. 92, 088302 (2004).

${ }^{17}$ H. Sato, E. C. I. Enobio, M. Yamanouchi, S. Ikeda, S. Fukami, S. Kanai, F. Matsukura, and H. Ohno, Appl. Phys. Lett. 105, 062403 (2014).

${ }^{18}$ N. W. Ashcroft and N. D. Mermin, Solid State Physics (Saunders College, New York, 1976), p. 708, ISBN-13: 978-0030839931.

${ }^{19}$ M. D. Kuz'min, Phys. Rev. Lett. 94, 107204 (2005).

${ }^{20}$ M. Charilaou, C. Bordel, P.-E. Berche, B. B. Maranville, P. Fischer, and F. Hellmano, Phys. Rev. B 93, 224408 (2016).

${ }^{21}$ S. Hashimoto, Y. Ochiai, and K. Aso, J. Appl. Phys. 67, 2136 (1990).

${ }^{22}$ D. Weller, Y. Wu, J. Stöhr, and M. G. Samant, Phys. Rev. B 49, 12888 (1994).

${ }^{23}$ M. J. Donahue and D. G. Porter, "OOMMF User's Guide, Version 1.0," Interagency Report NISTIR 6376, National Institute of Standards and Technology, Gaithersburg, MD (Sept 1999), see http://math.nist.gov/ oommf/.

${ }^{24}$ D. H. Lee and S. H. Lim, Appl. Phys. Lett. 92, 233502 (2008).

${ }^{25}$ S. Van Beek, K. Martens, P. Roussel, Y. C. Wu, W. Kim, S. Rao, J. Swerts, D. Crotti, D. Linten, G. S. Kar, and G. Groeseneken, AIP Adv. 8, 055909 (2018). 